\title{
Start-Up Acceleration of Quartz Crystal Oscillator Using Active Inductance Double Resonance and Embedded Triggering Circuit
}

\author{
Tomio Sato ${ }^{1}$, Akira Kudo ${ }^{2}$, Tetsuya Akitsu ${ }^{1^{*}}$ \\ ${ }^{1}$ Department of Human, Environment and Medical Engineering, Interdisciplinary Graduate School of Medicine \\ and Engineering, University of Yamanashi, Kofu, Japan \\ ${ }^{2}$ SEIKO EPSON Corporation, Suwa, Japan \\ Email: ${ }^{*}$ akitsu@yamanashi.ac.jp
}

Received 3 February 2015; accepted 20 February 2015; published 26 February 2015

Copyright (C) 2015 by authors and Scientific Research Publishing Inc.

This work is licensed under the Creative Commons Attribution International License (CC BY). http://creativecommons.org/licenses/by/4.0/

(c) () OP

\begin{abstract}
Low-frequency double-resonance quartz crystal oscillator was developed with active inductance circuit aiming the start-up of stable oscillation of tuning fork-type quartz crystal resonator at $32.768 \mathrm{kHz}$ within $0.37 \mathrm{~ms}$. The initial oscillation is triggered by a part of crystal oscillator forming a CR oscillator. The negative resistance ranges to $4 \mathrm{M} \Omega$ at $g_{m f}$ of $4.1 \mu \mathrm{A} / \mathrm{V}$. In a limited frequency range, the circuit shows negative reactance $C_{c c i}=-3.4 \mathrm{pF}$ equivalent to inductance $L_{c c}=9.8 \mathrm{H}$. The Allan standard deviation indicated $10^{-11}$ to $10^{-10}$, showing high stability comparable to general quartz crystal oscillator.
\end{abstract}

Keywords

Double Resonance Quartz Crystal Oscillator, Active Inductance

\section{Introduction}

Piezoelectric quartz crystal oscillators have widely expanded in sensing of the environmental data such as static pressure and temperature. Acceleration of the piezoelectric oscillator enables the intermittent operation of the piezoelectric sensor for power management. Engineering issue in the acceleration of the start-up of low frequency quartz crystal oscillator includes 1) triggering circuit, 2) generation of large negative resistance, and 3) linearity of the active device in large amplitude oscillation. In this work, we aim at the acceleration of the startup of low frequency, tuning fork quartz crystal resonator within several oscillation periods, which enables the intermittent operation of the sensor system. Acceleration of the start-up is studied by the gain control in the 
quartz crystal oscillator using a cascade circuit in the frequency region of several Mega Hertz [1]-[3]. In recent works, double-resonance quartz crystal oscillator was reported for the enhancement of the frequency pulling [4] and the mode separation of the multimode quartz crystal resonator [5]. Stability of the oscillation frequency is generally discussed based on the moving average of the variance determined for the discrete samples following the proto call [6]-[8]. The modified Allan standard deviation for moving average of finite length data $(\mathrm{k}=10)$ was employed as the measuring rule of the short range stability.

\section{Design and Analysis of Quartz Crystal Oscillator}

\subsection{Acceleration of the Start-Up of a Quartz Crystal Oscillator}

Figure 1 shows a circuit diagram of the active inductance double resonance oscillator circuit. The initial oscillation is generated by a part this oscillator acting as a CR oscillator, and after the oscillation of the quartz crystal resonator current starts, the double resonance is established between the quartz crystal and an active inductance combined with the parallel capacitance resulting in the generation of negative resistance. Essential circuit constants $R_{2}, C_{4}$, and $C_{0}$ satisfy this resonance condition, where $C_{0}$ is the parallel capacitance of the quartz crystal resonator. $R_{2}$ settles the bias in the initial stage of the oscillation. $C_{4}$ stores the ground potential at the activation of the $V_{c c}$ voltage, inserted between the node connecting two inverters. The oscillation frequency is determined by a recharging-time constant $R_{2}$ multiplied by $C_{4}$. Capacitors $C_{2}$ and $C_{3}$ are load capacitors which is necessary for the generation of negative resistance. $C_{5}$ and $C_{6}$ are pass-capacitors between the bus-line and the circuit ground. $C_{0}$ and $C_{1}$ are reserved for the parallel capacitance of the resonator and the series capacitor of the motion $\mathrm{arm}$. The maximum negative resistance is generated at specified value of the conductance $g_{m f}$ of the active circuits: CMOS (Complementary Metal Oxide Semiconductor) inverters $\mathrm{IC}_{1}$ and $\mathrm{IC}_{2}$. The conductance is controlled by negative feedback resistors $R_{f}=R_{3}, R_{4}, R_{5}$, and $R_{6}$.

$$
g_{m f}=\frac{g_{m}}{1+R_{f} g_{m}} .
$$

Figure 2 shows simplified equivalent circuit-1. CMOS inverter $\mathrm{IC}_{1}$ and $\mathrm{IC}_{2}$ is replaced by two current sources controlled by the gate voltage $V_{\text {in }}$ and $V_{g}$.

Applying Kirchhoff's law, the relations for $I_{\text {out }}$ and $V_{\text {in }}$ are found. $V_{\text {in }}$ is the input voltage of $\mathrm{IC}_{1}$ and $I_{\text {out }}$ is the output current of $\mathrm{IC}_{2}$.

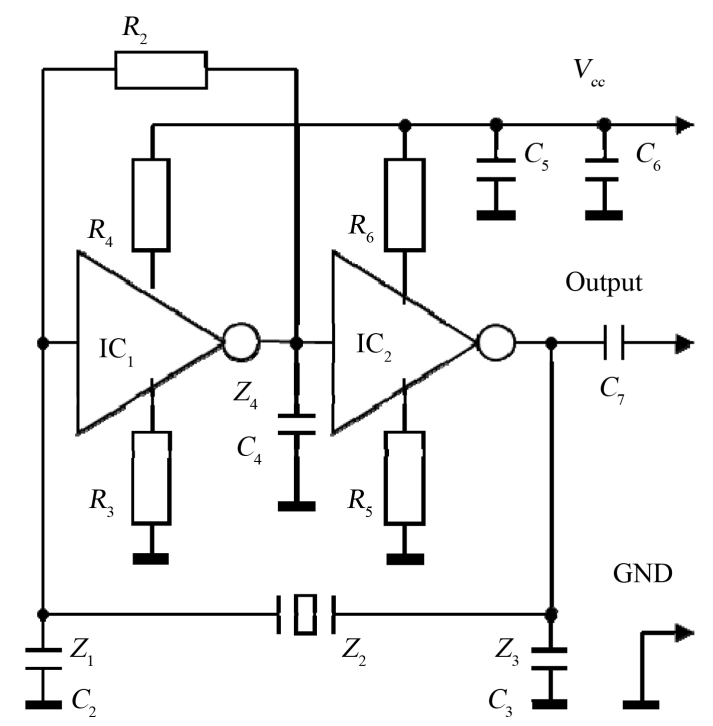

Figure 1. Circuit diagram of the quartz crystal oscillator. Circuit constants: Bias resistors $R_{3}, R_{4}, R_{5}$ and $R_{6}=3.3 \mathrm{k} \Omega$, feedback resistor $R_{2}=3 \mathrm{M} \Omega$, varied for the optimum setting; $C_{2}, C_{3}$, and $C_{4}=10 \mathrm{pF} ; C_{5}=0.1 \mu \mathrm{F} ; C_{6}=10 \mu \mathrm{F} ; C_{7}=100 \mathrm{pF}$; Inverter $\mathrm{IC}_{1}$ and $\mathrm{IC}_{2}$ TC7SHU04F; $V_{c c}=3 \mathrm{~V}$. Equivalent circuit constant of the quartz crystal resonator: $f_{1}=$ $32.768 \mathrm{kHz} ; L_{1}=11,797 \mathrm{H} ; R_{1}=47.6 \mathrm{k} \Omega ; C_{1}=2 \mathrm{fF}$. 


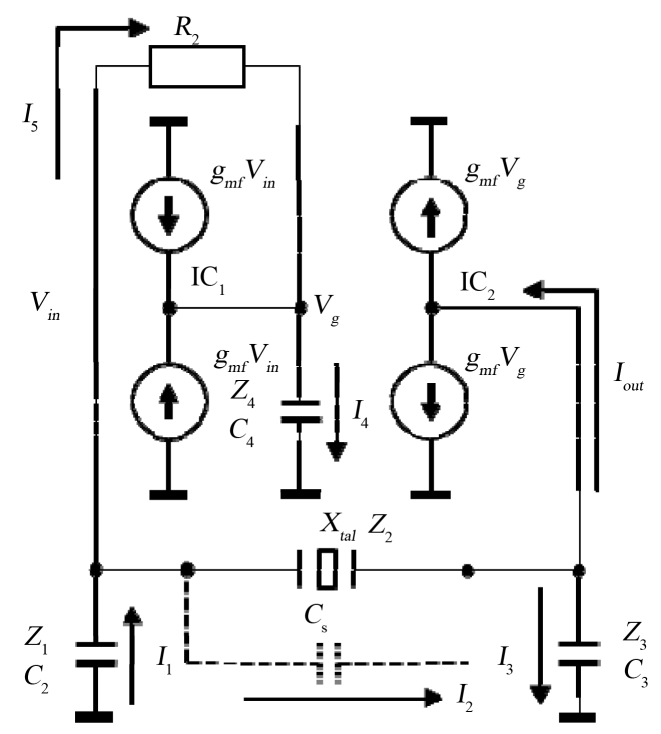

Figure 2. Simplified circuit: equivalent circuit-1.

$$
\begin{gathered}
I_{\text {out }}=2 g_{m f} Z_{4}\left(I_{5}+2 g_{m f} V_{\text {in }}\right)=2 g_{m f} V_{g}=2 g_{m f} Z_{4} I_{4} \\
V_{\text {in }}+R_{2} I_{5}+Z_{4} I_{4}=0 \text {, where } I_{4}=I_{5}+2 g_{m f} V_{\text {in }} . \\
I_{5}=-\frac{1+2 g_{m f} Z_{4}}{R_{2}+Z_{4}} V_{i n} . \\
I_{\text {out }}=2 g_{m f} Z_{4}\left(-\frac{1+2 g_{m f} Z_{4}}{R_{2}+Z_{4}}\right) V_{\text {in }}+4 g_{m f}^{2} Z_{4} V_{\text {in }} .
\end{gathered}
$$

Solving for the relation between $I_{\text {out }}$ and $V_{i n}$, total conductance $G_{M}$ is found.

$$
G_{M}=\frac{I_{\text {out }}}{V_{\text {in }}}=\frac{2 g_{m f}\left(2 g_{m f} R_{2}-1\right)}{1+\frac{R_{2}}{Z_{4}}} .
$$

Then the following relation is found. Current $I_{2}, I_{3}$ are expressed in the terms of $I_{1}$.

$$
\begin{gathered}
V_{\text {in }}=Z_{1} I_{1} . \\
I_{2}=I_{1}-I_{5}=\left(1+\frac{1+2 g_{m f} Z_{4}}{R_{2}+Z_{4}} Z_{1}\right) I_{1} . \\
I_{3}=I_{2}-I_{\text {out }}=I_{2}-G_{M} Z_{1} I_{1}=\left(1+\frac{1+2 g_{m f} Z_{4}}{R_{2}+Z_{4}} Z_{1}-G_{M} Z_{1}\right) I_{1} .
\end{gathered}
$$

Rearranging the expression, relation (11) is found.

$$
\begin{gathered}
Z_{1}+Z_{3}-G_{M} Z_{1} Z_{3}=Z_{c}, \quad Z_{c}=R_{c}+\frac{1}{j \omega C_{c}} . \\
Z_{2}+\frac{\left(\frac{R_{2}}{Z_{4}}+1\right) Z_{c}+\left(\frac{1}{Z_{4}}+2 g_{m f}\right) Z_{1} Z_{3}}{\frac{R_{2}}{Z_{4}}+1+\left(\frac{1}{Z_{4}}+2 g_{m f}\right) Z_{1}}=0 .
\end{gathered}
$$


$Z_{2}$ is the impedance of a quartz crystal resonator $\left(Z_{x t}\right)$, and impedance for other components is defined as in (12). The composed impedance $Z_{c c}$ of the active circuit is found, substituting the impedance. From the condition for the non-zero solution of current, the oscillation condition results in (13). The impedance of the circuit is divided into resistive and reactance parts.

$$
\begin{gathered}
Z_{2}=Z_{x t}, Z_{1}=\frac{1}{j \omega C_{2}}, Z_{3}=\frac{1}{j \omega C_{3}}, Z_{4}=\frac{1}{j \omega C_{4}}, \omega_{r}=\frac{1}{C_{4} R_{2}} . \\
Z_{x t}+Z_{c c}=0 \\
Z_{c c}=R_{c c}+j X_{c c}=\frac{R_{c}+\frac{1}{\omega_{r} C_{c}}-\frac{2 g_{m f}}{\omega^{2} C_{2} C_{3}}+j\left(\frac{\omega R_{c}}{\omega_{r}}-\frac{C_{4}}{\omega C_{2} C_{3}}-\frac{1}{\omega C_{c}}\right)}{1+\frac{C_{4}}{C_{2}}+j\left(\frac{\omega}{\omega_{r}}-\frac{2 g_{m f}}{\omega C_{2}}\right)} .
\end{gathered}
$$

The equivalent resistance and the reactance of the circuit are found. Equivalent inductance $L_{c c}$ or capacitance $C_{c c}$ is determined depending on sign of reactance $X_{c c}$.

$$
R_{c c}=\frac{a c+b d}{a^{2}+b^{2}}, X_{c c}=\frac{a d-b c}{a^{2}+b^{2}},\left(L_{c c}=\frac{X_{c c}}{\omega}, C_{c c}=-\frac{1}{\omega X_{c c}}\right) .
$$

Factors "a", "b", "c" and "d" are introduced for the simplicity of the expression, where factors "c" and "d" have the dimension of $\Omega$ and factors " $a$ " and " $b$ " are dimensionless numbers.

$$
a=1+\frac{C_{4}}{C_{2}}, b=\frac{\omega}{\omega_{r}}-\frac{2 g_{m f}}{\omega C_{2}}, c=\frac{1}{\omega_{r} C_{c}}+R_{c}-\frac{2 g_{m f}}{\omega^{2} C_{2} C_{3}}, d=\frac{\omega R_{c}}{\omega_{r}}-\frac{C_{4}}{\omega C_{2} C_{3}}-\frac{1}{\omega C_{c}} .
$$

$G_{M}$ is separated into real and imaginary parts.

$$
\begin{gathered}
G_{M}=g_{s}+j b_{s} . \\
g_{s}=\frac{2 g_{m f}\left(2 g_{m f} R_{2}-1\right)}{1+\left(\frac{\omega}{\omega_{r}}\right)^{2}}, b_{s}=\frac{\omega}{\omega_{r}} \frac{2 g_{m f}\left(1-2 g_{m f} R_{2}\right)}{1+\left(\frac{\omega}{\omega_{r}}\right)^{2}} .
\end{gathered}
$$

Introducing (13) and (19) into $Z_{c c}$, the impedance of the active circuit is found.

$$
R_{c c}=\frac{g_{s}}{\omega^{2} C_{2} C_{3}}, \frac{1}{C_{c c}}=\frac{1}{C_{2}}+\frac{1}{C_{3}}-\frac{b_{s}}{\omega C_{2} C_{3}} .
$$

Figure 3 shows simplified diagram of equivalent circuit- 2 of the oscillator.

The active circuit is indicated with $R_{c c}$ and reactance $C_{c c}$ or $L_{c c}$ depending on the sign. The resonator consists of parallel capacitance $C_{0}$ and the motion arm, $L_{1}, C_{1}$, and $R_{1}$, the equivalent series inductor, capacitor, and resistor respectively. $C_{S}$ is a stray capacitance. Calculating the parallel composition of $C_{0}$ and $C_{s}$ with the active circuit, equivalent circuit-3 in Figure 4 is found. Composed equivalent resistance $R_{c c i}$ and capacitance $C_{c c i}$ are found.

$$
R_{c c i}=\frac{R_{c c}}{\left(1+\frac{C_{0 s}}{C_{c c}}\right)^{2}+\left(\omega C_{0 s} R_{c c}\right)^{2}}, \frac{1}{C_{c c i}}=\frac{\frac{1}{C_{c c}}\left(1+\frac{C_{0 s}}{C_{c c}}\right)+\frac{\left(\omega C_{0 s} R_{c c}\right)^{2}}{C_{0 s}}}{\left(1+\frac{C_{0 s}}{C_{c c}}\right)^{2}+\left(\omega C_{0 s} R_{c c}\right)^{2}} .
$$

Negatively signed capacitance is converted to an active inductance by relation (21),

$$
L_{c c i}=-\frac{1}{\omega^{2} C_{c c i}} \text {. }
$$




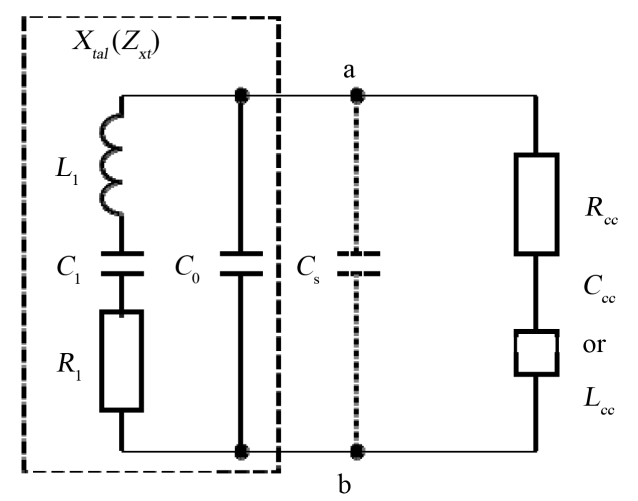

Figure 3. Equivalent circuit-2.

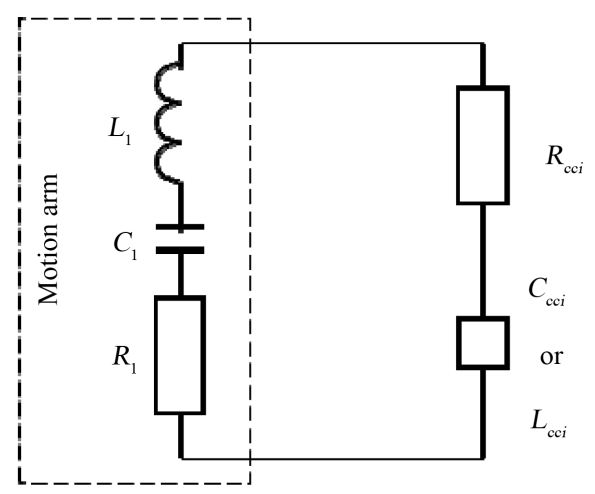

Figure 4. Equivalent circuit-3.

The denominator of negative resistance $R_{c c i}$ has quadratic dependence on $R_{c c}$. The maximum value of the absolute value is reached at a specific value of $R_{c c}$ determined by $C_{0 s}$ and $C_{c c}$. The following relation is fulfilled.

$$
\left|R_{c c i}\right|_{\max }=\frac{1}{2 \omega C_{0 s}\left(1+\frac{C_{0 s}}{C_{c c}}\right)}, \text { at }\left|R_{c c}\right|=\frac{1}{\omega C_{0 s}}\left(1+\frac{C_{0 s}}{C_{c c}}\right) .
$$

The active inductance appears in the vicinity of the resonance frequency, while capacitance $C_{c c}$ is negative. The resonance frequency is determined by $L_{c c}, C_{0 s}$, and the sum of $C_{0}$ and $C_{s}$. In this simplified form, the absolute value of negative resistance $R_{c c i}$ becomes infinitely large, if $C_{c c}$ approaches- $C_{0 s}$ and condition (23) is fulfilled.

$$
C_{c c}=-\frac{1}{\omega^{2} L_{c c}} .
$$

At the resonance frequency determined by $L_{c c}$ and $C_{0 s}$, the absolute value of negative resistance determines the growth of signal. The suppression of negative resistance by inductance $L_{1}$ establishes the stability and inhibitory action against the signal growth. Table 1 shows the equivalent circuit constant of the quartz crystal resonator.

Figure 5 compares the absolute value of negative resistance $R_{c c i}$ for parameters $L_{c c}$ and $C_{c c}$. Figure 6 shows the absolute value of negative resistance $R_{c c}$ as functions of frequency and $g_{m f}$. The enhancement of negative resistance and the correlation with active inductance is explained in the following part. Resonance occurs in the inductive region of the motion arm, while the reactance of the active circuit is capacitive. The maximum absolute value of negative resistance approximately $1.3 \mathrm{M} \Omega$ is obtained with infinitely large $C_{c c}$. The maximum value is limited at $0.8 \mathrm{M} \Omega$ for $C_{c c}=5 \mathrm{pF}$, a practical value. Larger negative resistance is generated in the region where the active circuit is inductive. When the circuit reactance is inductive, the maximum value increases. The maximum absolute value of negative resistance approximately $13 \mathrm{M} \Omega$ for $C_{c c i}=-2 \mathrm{pF}$ equivalent to active 
Table 1. Equivalent circuit constant of the quartz resonator.

\begin{tabular}{cccccc}
\hline \multirow{2}{*}{ Quartz resonator } & \multicolumn{5}{c}{$\tau(\mathrm{ms})$} \\
\cline { 2 - 6 } & $L_{1}$ & $C_{1}$ & $R_{1}$ & $C_{0}$ & $Q_{1}$ \\
\hline Tuning fork-type $(32.768 \mathrm{kHz})$ & $11,797 \mathrm{H}$ & $2 \mathrm{fF}$ & $47.6 \mathrm{k} \Omega$ & $1.14 \mathrm{pF}$ & 51,023 \\
\hline
\end{tabular}

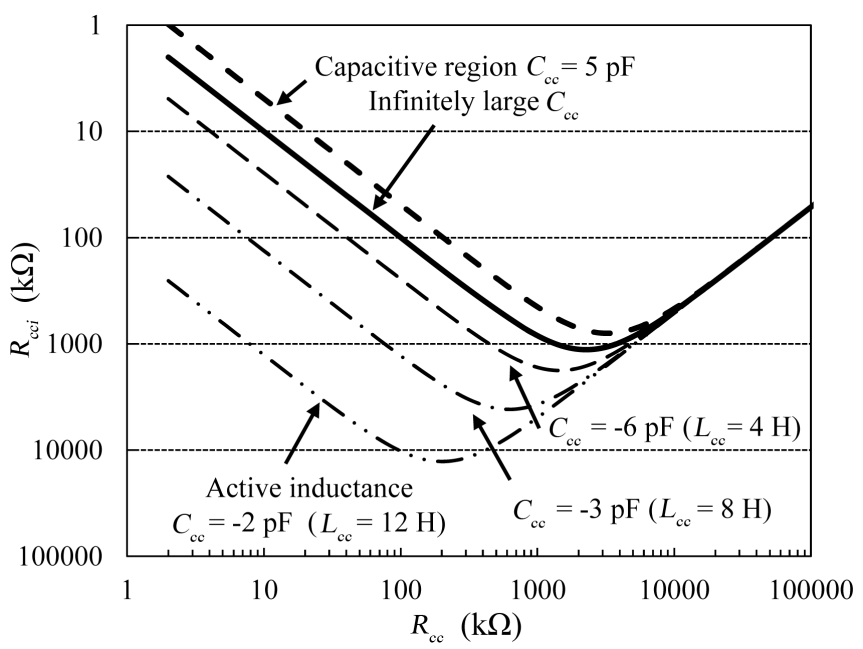

Figure 5. Comparison of the absolute values of negative resistance for $L_{c c}$ and $C_{c c}$, as functions of the negative resistance $R_{c c}$. Circuit constants: $C_{0}=1.14 \mathrm{pF} ; C_{S}=1 \mathrm{pF}$.

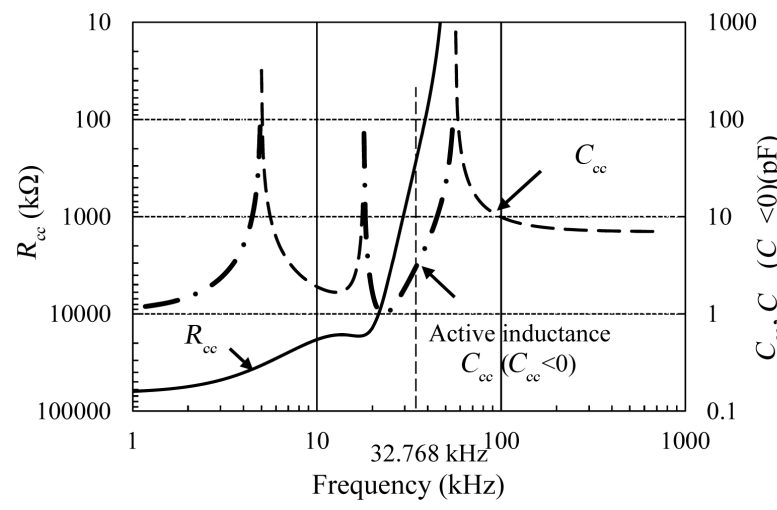

(a)

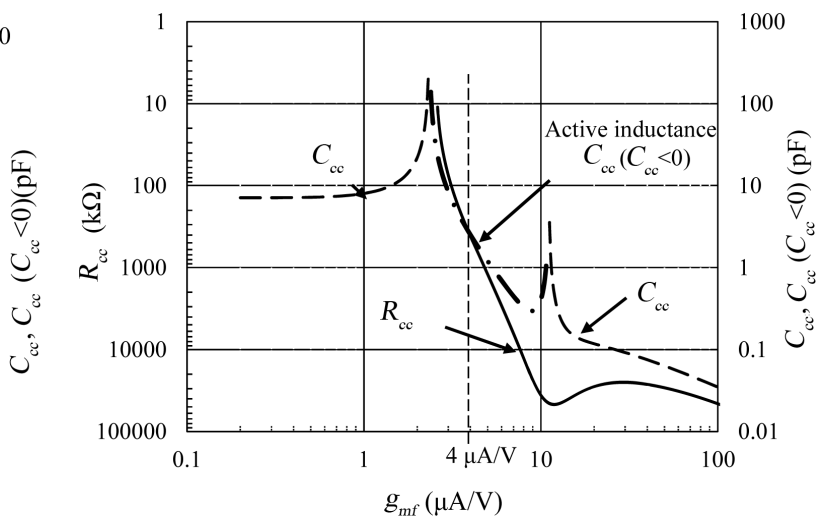

(b)

Figure 6. Absolute value of negative resistance $R_{c c}$ and reactance $C_{c c}$ as functions of frequency and gain. Circuit constant: $C_{2}$ and $C_{3}=14 \mathrm{pF} ; C_{4}=18 \mathrm{pF} ; R_{2}=1.0 \mathrm{M} \Omega$, (a) $g_{\mathrm{mf}}=4 \mu \mathrm{A} / \mathrm{V}$, (b) $f_{r}=32.768 \mathrm{kHz}$.

inductance $L_{c x}=12 \mathrm{H}$.

In Figure 6(a), parameter $g_{m f}$ is selected at $4 \mu \mathrm{A} / \mathrm{V}$. At the lower gain, $g_{m f}=2 \mu \mathrm{A} / \mathrm{V}$, the active inductance appears in the lower frequency region and disappears in the higher frequency region. For example, it appears at $10 \mathrm{kHz}$ and disappears at $27 \mathrm{kHz}$. The frequency limit is $55 \mathrm{kHz}$ for $g_{m f}=4 \mu \mathrm{A} / \mathrm{V}$, and $110 \mathrm{kHz}$ for $g_{m f}=8 \mu \mathrm{A} / \mathrm{V}$. In the lower frequency region, at approximately $9 \mathrm{kHz}$, the active inductance disappears. Figure 6(b) shows the dependence on conductance $g_{m f}$. At frequency of $32.768 \mathrm{kHz}$, the active inductance appears for $g_{m f}=2.4 \mu \mathrm{A} / \mathrm{V}$. The limit varies depending on the oscillation frequency. The frequency limit is $20 \mathrm{kHz}$ for $g_{m f}=1.5 \mu \mathrm{A} / \mathrm{V}, 40$ $\mathrm{kHz}$ for $g_{m f}=3 \mu \mathrm{A} / \mathrm{V}$ and $110 \mathrm{kHz}$ for $g_{m f}=8 \mu \mathrm{A} / \mathrm{V}$. The negative resistance and the active inductance appear from the low frequency side, and the resonance condition with CR oscillation is established before the motion arm appears.

$$
C_{0}-C_{c c}=0
$$


Figure 7 shows the dependence of the absolute value of negative resistance $R_{c c i}$ on frequency and $g_{m f}$. Figure 7(a) shows the frequency dependence of $R_{c c i}$ and reactance $L_{c c i}, C_{c c i}$. The maximum value of negative resistance $R_{c c i}$ ranges to $4 \mathrm{M} \Omega$. This result is obtained in the case of inductive reactance $L_{c c i}=9.8 \mathrm{H}$. The composed reactance is capacitive $C_{c c i}=3.4 \mathrm{pF}$. Figure 7(b) shows the dependence of the absolute value of negative resistance $R_{c c i}$ and reactance $C_{c c i}$ on $g_{m f}$. For $g_{m f}=4.1 \mu \mathrm{A} / \mathrm{V}$, the absolute value of negative resistance is $4 \mathrm{M} \Omega$. The composed circuit reactance $C_{c c i}=-3.4 \mathrm{pF}$ is equivalent to $L_{c c}=9.8 \mathrm{H}$.

Figure 8 shows the absolute value of negative resistance for different resonance frequency. This result tells that the active inductance is generated with certain value of gain in a narrow range corresponding to the required frequency. This result indicates that high gain is not necessarily for better performance.

This result suggests a design principle of the circuit: Higher resonance frequency needs higher $\mathrm{g}_{m f}$. In this analysis, we take a look at the circuit impedance from the resonator terminal. The parallel capacitance $C_{0}$ and stray capacitance $C_{s}$ are included in the impedance of the active circuit. Thus, the relation between $R_{c c i}$ and $R_{c c}$ is presented, As a part of the final solution, the result that $R_{c c i}$ becomes infinitely large at $C_{c c}=-C_{0 s}$, must be interpreted carefully in the context of the actual circuit design. The minimum idea given here is that the active inductance can generate large negative resistance compared to the capacitive region. Actually, $R_{c c}$ is determined by number of circuit constants and angular frequency of the oscillation, and the strength of the oscillation is limited within the linear region of the active circuit.

\subsection{Modelling of the Start-Up of Initial Oscillation}

The crystal current through the motion arm is not generated in the initial stage of the oscillation. In another

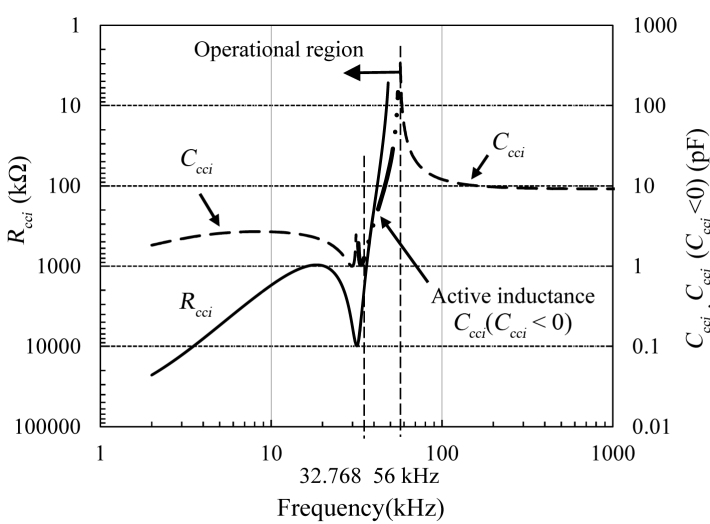

(a)

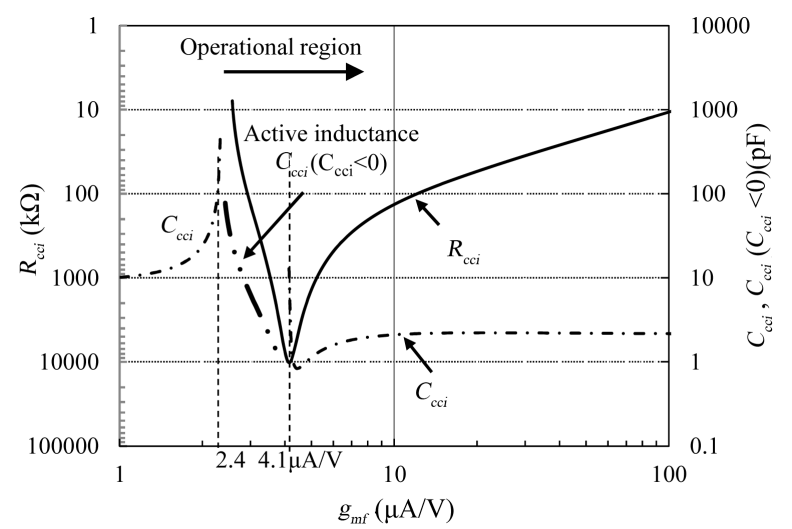

(b)

Figure 7. Absolute value of negative resistance $R_{c c i}$ and reactance as functions of frequency and $g_{m f}$. Ciruit constant: $R_{2}=1.9$ $\mathrm{M} \Omega ; C_{2}$ and $C_{3}=14 \mathrm{pF} ; C_{0}=1.14 \mathrm{pF} ; C_{4}=18 \mathrm{pF} ; C_{s}=1 \mathrm{pF}$. (a) $g_{m f}=4 \mu \mathrm{A} / \mathrm{V} ; C_{x}$ infinite. (b) $f_{r}=32.768 \mathrm{kHz}$.

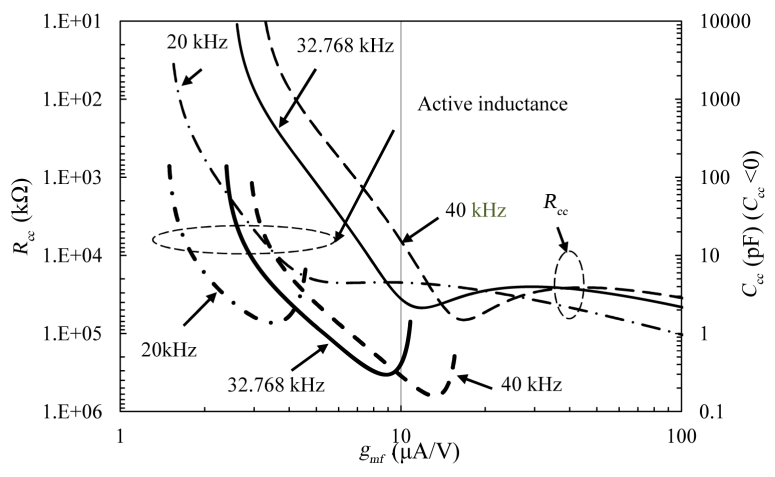

(a)

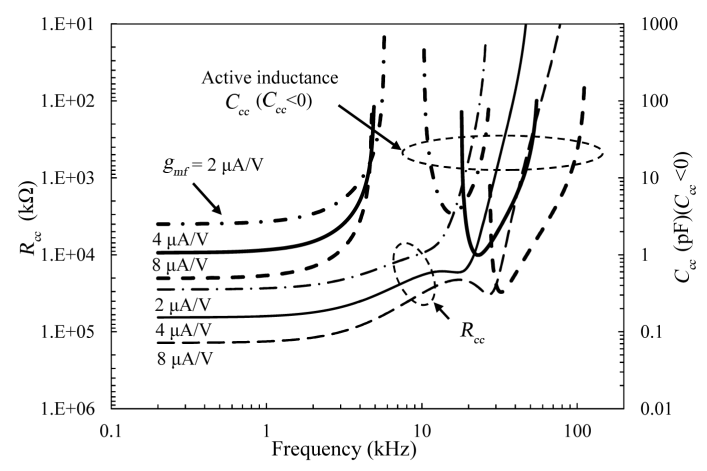

(b)

Figure 8. Comparison of negative resistance for different values of $g_{m f}$ and $f_{r}$. (a) Frequency $f_{r}=20,32.768$, and $40 \mathrm{kHz}$. Circuit constants: $C_{2}$ and $C_{3}=14 \mathrm{pF} ; C_{4}=18 \mathrm{pF} ; R_{2}=1.9 \mathrm{M} \Omega$. (b) $g_{m}=2,4$, and $8 \mu \mathrm{A} / \mathrm{V}$. Circuit constants: $C_{2}$ and $C_{3}=10$ $\mathrm{pF} ; C_{4}=18 \mathrm{pF} . R_{2}=1.9 \mathrm{M} \Omega$. 
expression, this branch does not exists in the circuit. Because of high Q, the start-up needs reasonable acceleration system. Figure 9 shows simplified circuit diagrams of the oscillation Mode- 1 and Mode- 2 . Before the establishment of the resonance oscillation, parallel capacitance $C_{0}$ is the existing circuit component and the motion arm is disconnected. Based on the result of analysis, the active circuit is indicated with $C_{c c i}$ and the composed equivalent negative resistance $R_{c c i}$. The oscillation frequency is determined by the equivalent reactance of the active circuit and $C_{0}$. The motion arm appears after the certain growth of the crystal current. This figure also explains the relation between $R_{c c}$ and $R_{c c i}$. The circuit in Figure 9(b) is closed with the motion arm, and oscillation current $i(t)$ is excited in the closed loop. $V_{x}$ and $V_{c}$ may have different initial values, frequency and polarity depending on the switching sequence.

The start-up mode of the oscillator depends on the rise of $V_{\text {cc }}$. When the bias current increases the CR oscillation as in Mode- 1 starts before the establishment of the crystal current. The oscillation frequency of Mode- 1 is determined by $R_{2}$ multiplied by the composed capacitance. When the quartz crystal resonator is activated sufficiently, the motion arm appears in the circuit, as in Model-2. Computer simulation was carried out using LTspice for Windows (Linear Technology Corporation, 1630 McCarthy Blvd., Milpitas, CA, USA) [9]. Figure 10 shows the transient excitation of the crystal current with matched frequency setting: the oscillation frequency of the CR oscillator is slightly higher than the resonance frequency. The crystal current in the motion arm grows up faster and the oscillation frequency of the entire oscillator circuit is locked to the resonance frequency.

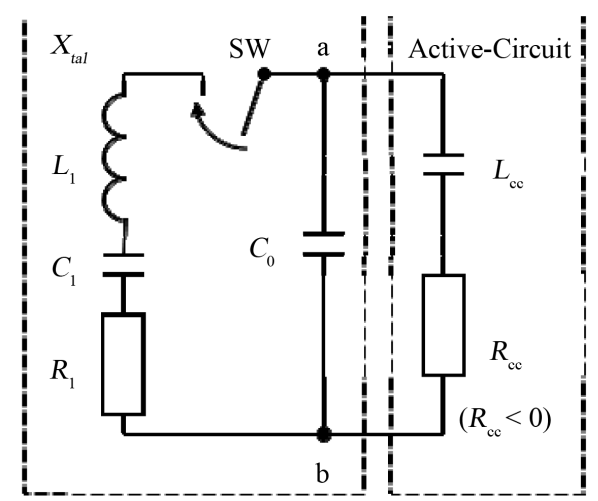

(a)

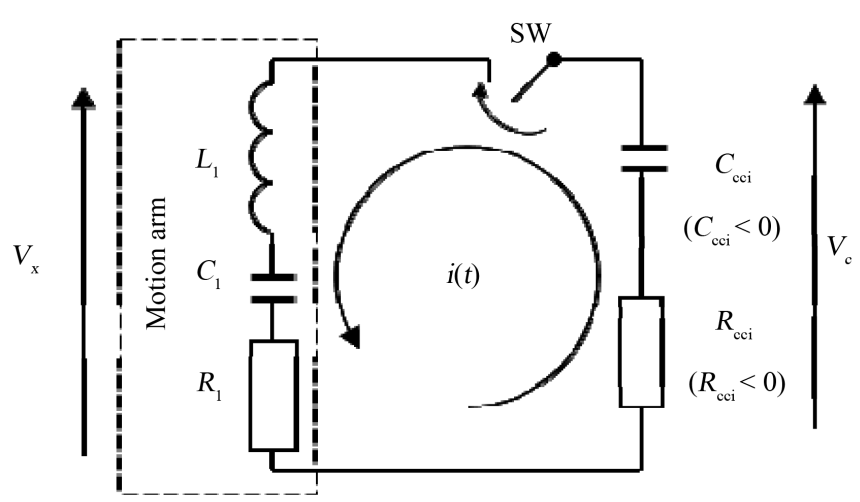

(b)

Figure 9. Equivalent circuit diagram of quartz crystal oscillator. (a) Model-1; (b) Model-2.

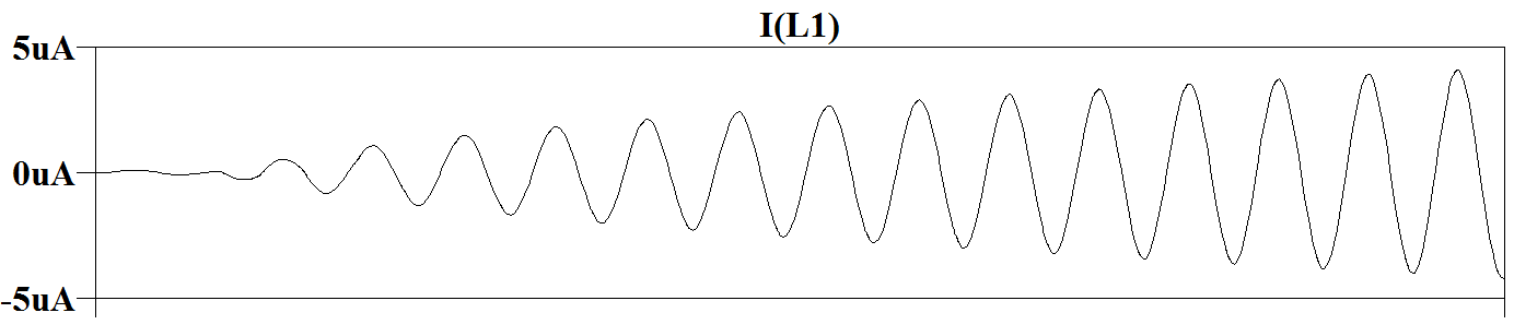

Ous

$500 u s$

V(out)

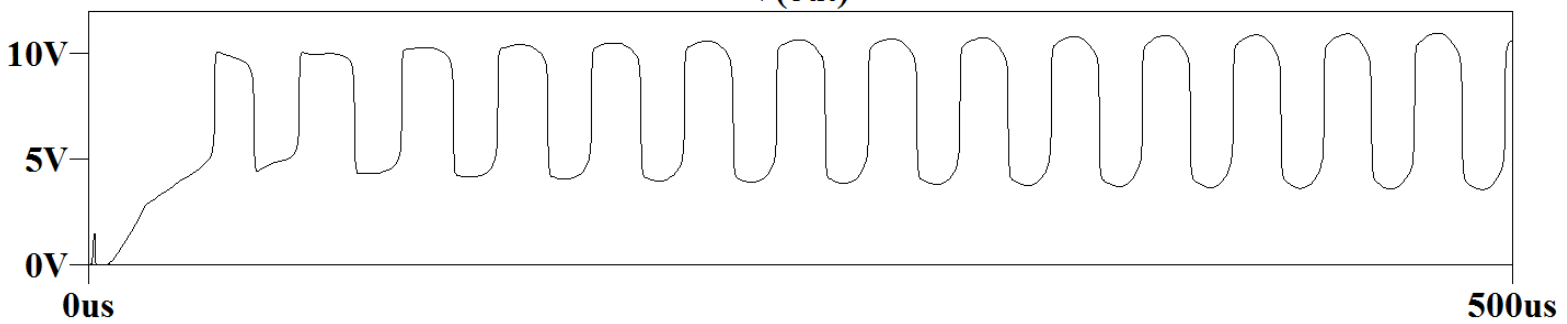

Figure 10. Initial stage with frequency match conditions. Upper track: Current $\mathrm{I}\left(L_{1}\right)$ fowing through the series inductance $L_{1}$. Lower track: Output voltage. 
Figure 11 shows the initial stage of the mismatched case. If the frequency of the CR oscillation is too high, and the oscillation apparently starts at different frequency. The oscillation frequency abruptly locked to the crystal resonance frequency by the growth of the crystal current. The faster growth of crystal resonance occurs with frequency mated pumping by the CR oscillation. The similar result of the matched and mismatched case was observed in the experiment.

Figure 12 shows the circuit diagram for the computer simulation. Here, CMOS inverter $\mathrm{IC}_{1}$ and $\mathrm{IC}_{2}$ are replaced with pairs of complementary MOSFETs (Metal Oxide Semiconductor Field Effect Transistor).

The circuit constants of the motion arm are not corresponding to the values assigned in the analysis and experiment. Also, the delayed connection of the motion arm is not considered in this simulation.

When the motion arm is removed, this circuit forms a CR oscillator. The oscillation frequency is determined by the reactance of the parallel capacitance of the quartz resonator and feedback resistor $R_{2}$. Figure 13 shows a typical wave form of the CR oscillator and FFT spectral analysis, for the matched frequency peak in the vicinity of the resonance frequency of the motion arm. The CR oscillation peak appears in the vicinity of the quartz resonance frequency.

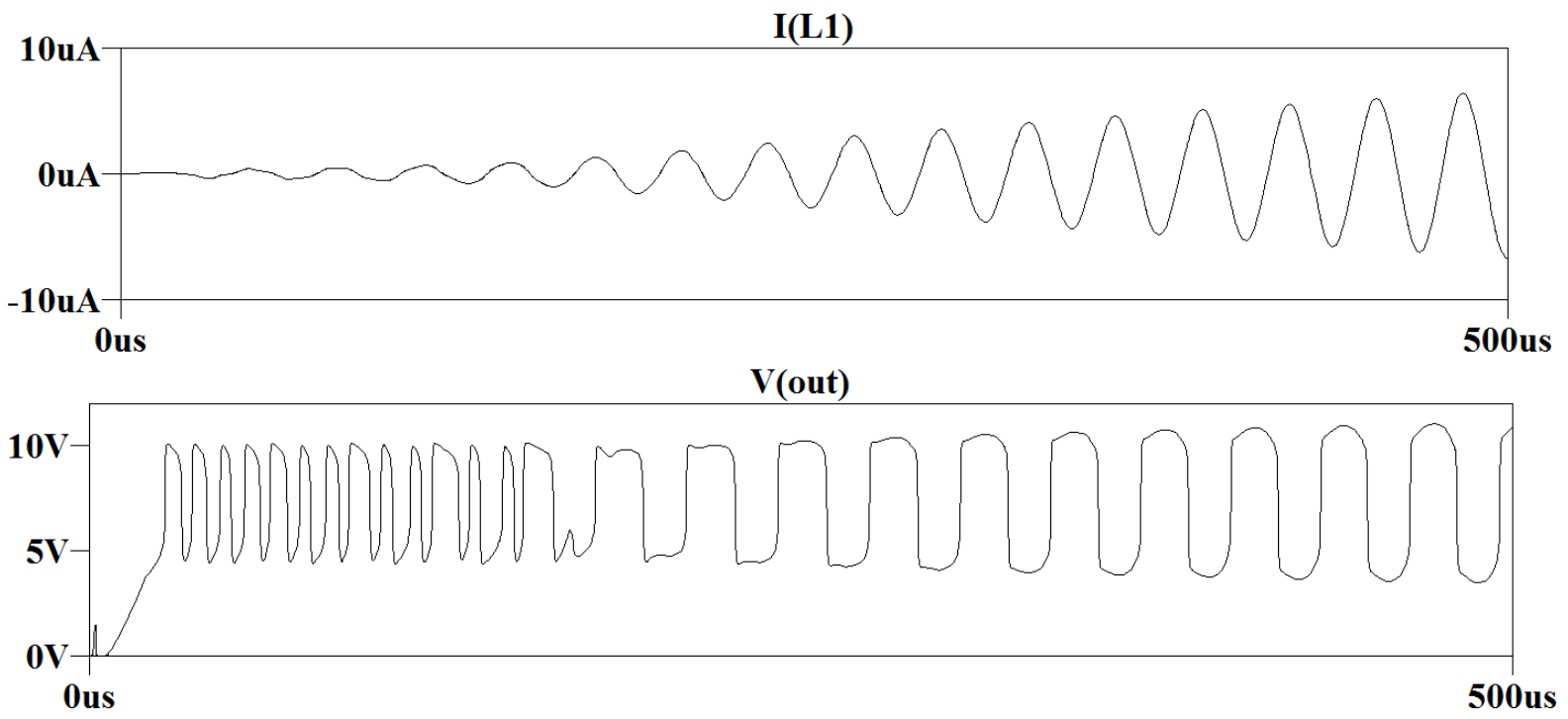

Figure 11. Comparison of growth of the crystal current and output with a mismatch conditions at $R_{2}=0.8 \mathrm{M} \Omega$. Upper track: Current $\mathrm{I}\left(L_{1}\right)$ flowing through the series inductance $L_{1}$; Lower track: Output voltage.

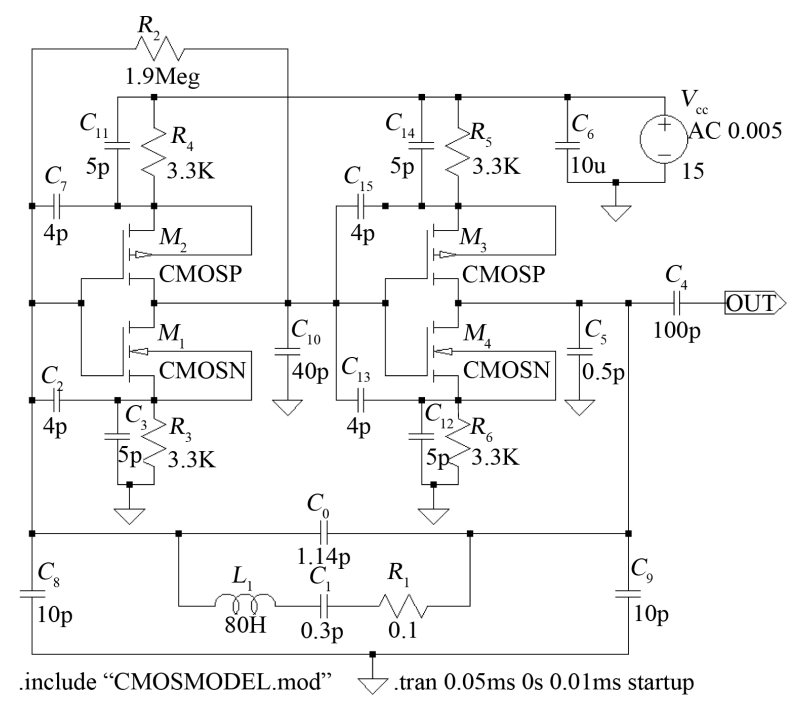

Figure 12. Equivalent circuit for the computer simulation. Courtesy of LTspice, Linear technology corporation. 


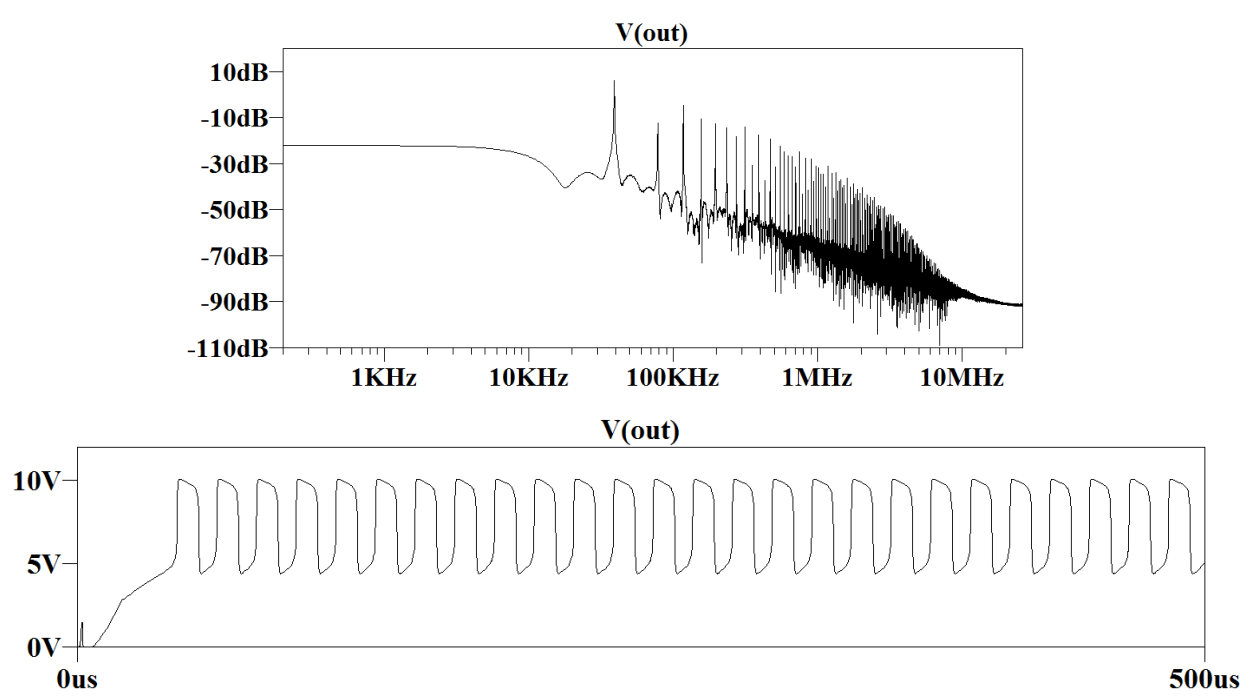

Figure 13. Typical example for the waveform of the CR oscillator and the FFT spectral analysis $R_{2}=3.0 \mathrm{M} \Omega$.

\section{Experimental Result and Discussions}

The start-up and the stability of the stable oscillation of the double resonance oscillator is experimentally evaluated. The stability of the oscillation frequency is analyzed with 53,230 A universal frequency counter (Agilent Technologies, Santa Clara, Ca, USA) synchronized with external rubidium oscillator with long period stability < $2 \times 10^{-11} /$ month and short period stability $<1 \times 10^{-11} / \mathrm{s}$.

\subsection{Start-Up of the Double Resonance Quartz Crystal Oscillator}

The proposed quartz oscillator circuit is activated with $V_{\mathrm{cc}}$ and the minimum start-up time marked $0.37 \mathrm{~ms}$, as shown in Figure 14. The start-up time and the CR oscillation frequency are shown as functions of the resistance $R_{2}$. In the proposed oscillator circuit, the output of inverter $\mathrm{IC}_{2}$ is positively fed back to the input of inverter $\mathrm{IC}_{1}$ through the quartz crystal resonator. Replacing the quartz resonator with a capacitor equivalent to the parallel capacitance $C_{0}$, the circuit exhibits CR oscillation and this frequency is determined by the time constant: $R_{2}$ multiplied by $C_{4}$. In the case of $R_{2}=2.3 \mathrm{M} \Omega$, the frequency of the CR oscillation $f_{C R}$ is approximately equal to the resonance frequency. The quartz crystal oscillation is triggered by the CR oscillation and the start-up time shows its minimum. The entire oscillator circuit is synchronized after short transient, showing the rapid start demonstrated with typical waveform: the initiation of the oscillation within $0.37 \mathrm{~ms}$, several oscillation cycle. Figure 15 shows a typical example for the accelerated start-up of the quartz crystal oscillator.

\subsection{Stability of the Double Resonance Quartz Crystal Oscillator}

In this experiment, modified two-sample Allan standard deviation is employed as a measure of the short-time frequency stability. This protocol is defined in (25), following IEEE Standard 1139 [9]. The frequency of oscillator circuit $f_{k}$ is the discrete sample of oscillation frequency. $\tau$ is the gate time and $n$ is the sequential number of samples. Dimensionless parameter is defined from frequency deviation normalized by the moving average over 10 sequential samples. Figure 16 shows the modified Allan standard deviation showing the short range stability of the order of $10^{-10}$ to $10^{-11}$. The quartz crystal oscillator was isolated in a shield box with isolated DC power supply. This result satisfies the industrial requirement for the standard quartz sensor.

$$
\sigma_{y}(\tau)=\left[\frac{2}{M-1} \sum_{k=1}^{M-1}\left(\frac{f_{k+1}-f_{k}}{f_{k+1}+f_{k}}\right)^{2}\right]^{\frac{1}{2}} .
$$

Table 2 shows the stability of the quartz crystal oscillation in this experiment. This result shows Allan standard 


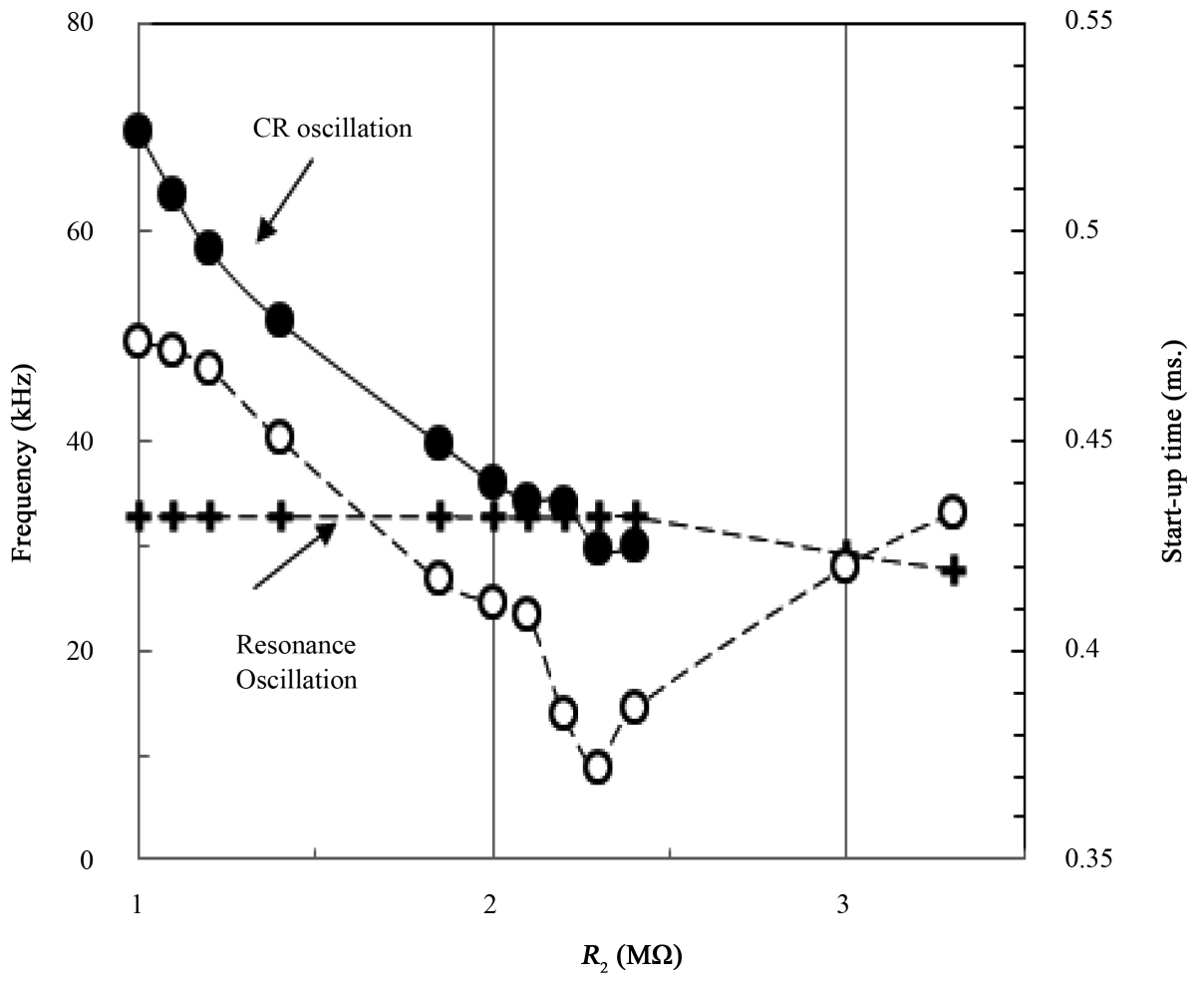

Figure 14. Experimental comparison of the start-up time and the frequency of the CR oscillation.

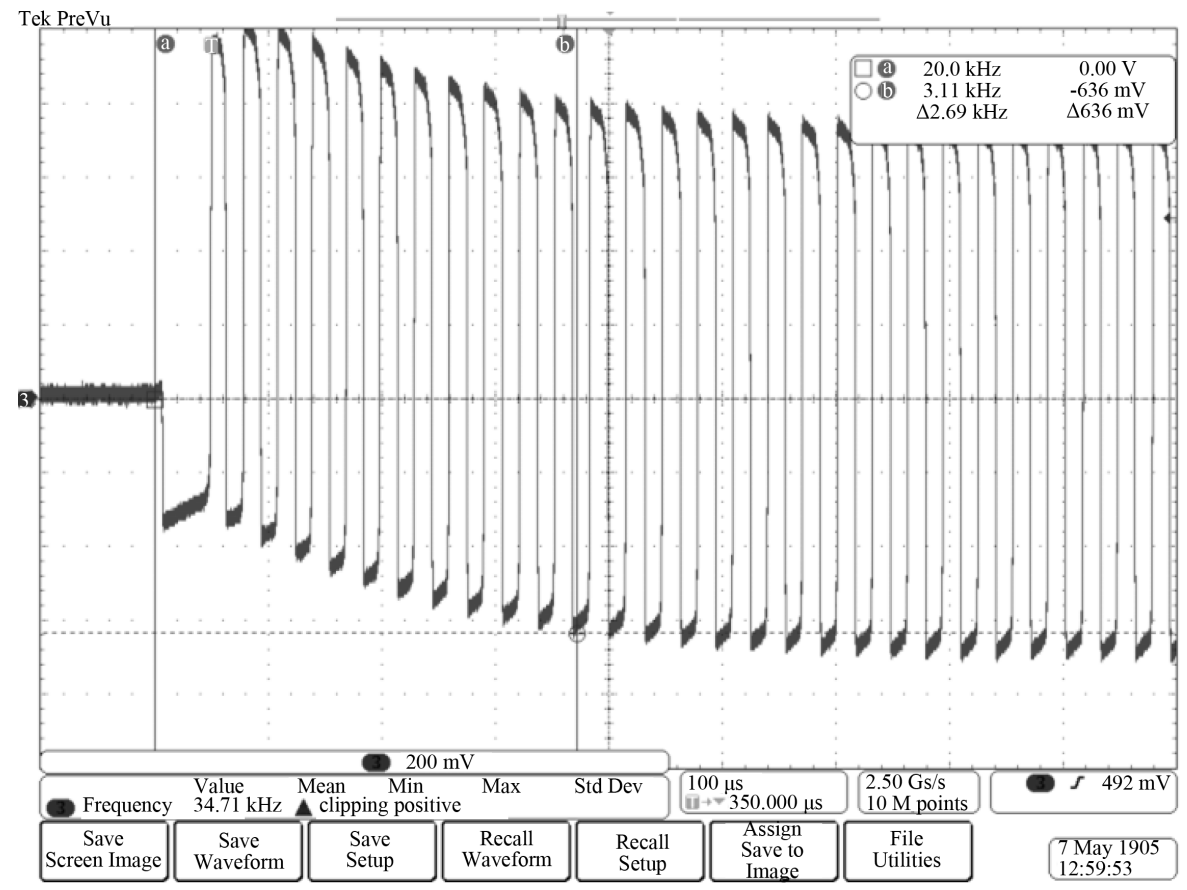

Figure 15. Start-up of the double-resonance quartz crystal oscillator. Horizontal scale: $100 \mu \mathrm{s} / \mathrm{div}$. Vertical scale $200 \mathrm{mV} /$ div. Circuit constants: $C_{2}, C_{3}$ and $C_{4}=10 \mathrm{pF}$. Start-up of the stable oscillation $0.37 \mathrm{~ms}$ after the activation of $V_{c c}$.

deviation of $10^{-11}$ satisfy the requirement for the standard sensing. Probably, for the further improvement of the stability, the improvement of the $Q$-value of the resonator is necessary. 


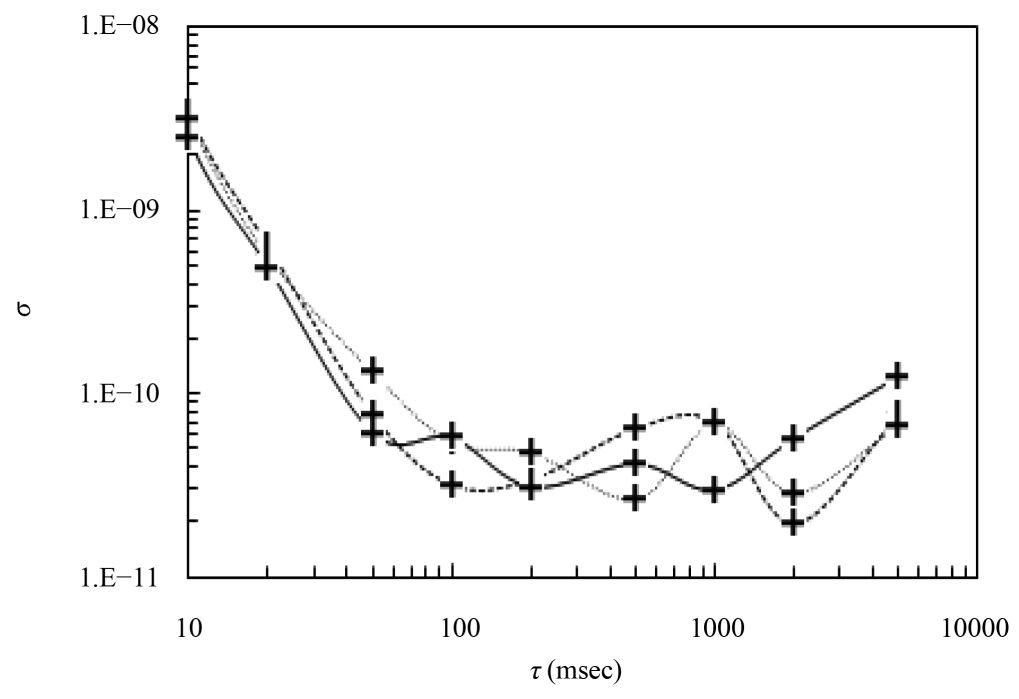

Figure 16. Short range stability of the double-resonance quartz crystal oscillator.

Table 2. Stability: Averaged Allan standard deviation of the proposed circuit.

\begin{tabular}{ccccc}
\hline \multirow{2}{*}{ Quartz oscillator circuit } & \multicolumn{3}{c}{$\tau(\mathrm{ms})$} \\
\cline { 2 - 5 } & 1 & 10 & 100 & 1000 \\
\hline Double-Resonance Quartz Oscillator & $2.7 \times 10^{-7}$ & $2.4 \times 10^{-8}$ & $2.7 \times 10^{-10}$ & $6.8 \times 10^{-11}$ \\
\hline
\end{tabular}

\section{Conclusion}

Environmental sensing awaits solutions to reduce the electric-power, in continuous monitoring. The quick start of quartz crystal oscillators allows excitation of stationary oscillation established after short transient meeting the request for the power management in the environmental sensing such as the pressure and temperature. In this work, we resolved the engineering issues for the rapid start-up: 1) Large negative resistance; 2) Low distortion and linearity; 3) Triggering circuit. The start-up of a low frequency quartz oscillator is triggered with a CR oscillator and transferred to a quartz crystal oscillator. The maximum negative resistance ranges to $4 \mathrm{M} \Omega$ at specified gain of the active CMOS inverter circuit $g_{m f}=4.1 \mu \mathrm{A} / \mathrm{V}$. The composed reactance of the active circuit $C_{c c i}$ shows negative value, $-3.4 \mathrm{pF}$ which acts as inductance of $9.8 \mathrm{H}$ and generates large negative resistance. Rapid start-up of the oscillation was established by the energy transfer by the initial CR oscillation of the active circuit and the minimum start-up time was realized. The oscillation condition was examined by the analysis and the start-up in the initial stage was examined by the computer simulation and experiment. The result shows corresponding dependence of the start-up time on circuit parameter $R_{2}$. The stability performance of the double-resonance oscillator showed that short range stability of $10^{-11}$ satisfies the industrial requirement for the standard quartz oscillator circuit.

\section{Acknowledgements}

The authors acknowledge Ms Ruzaini Izyan binti Ruslan and Mr. Satoshi Goto for their collaboration in the early stage of this experiment. This work was supported in part by JST A-STEP Contract No. AS251Z01794J.

\section{References}

[1] Izumiya, S., Asaki, J. and Adachi, T. (2003) Algebraic Analysis Method of Start-Up Characteristics of Cascade Crystal Oscillator. Electronics and Communications in Japan (Part II: Electronic), 86, 32-43. http://dx.doi.org/10.1002/ecjb.10166

[2] Ulmer, R.W. (1990) Stat-Up Circuit for Low Power MOS Crystal Oscillator. US Patent 4956618.

[3] Lu, W., Tse, T. and Chan, W.-C. (2006) Crystal Oscillator Circuit Having a Start-Up Time Reduction Circuit. US Patent 7034628 B1. 
[4] Ehara, K., Tanaka, H., Sato, T. and Kanno, Y. (2006) Development of Inductive Quartz Crystal Oscillator Circuit with CMOS Inverter. Proceedings of IEEE Conference on System, Man and Cybernetics, Taipei, 8-11 October 2006, 14251430.

[5] Izyan-Ruslan, R., Sato, T. and Akitsu, T. (2012) Voltage Controlled Narrow Band Wide-Variable Range Four Segment Quartz Crystal Oscillator. IEEE Transactions on Ultrasonics, Ferroelectrics, and Frequency Control, 59, 564-572.

[6] Barnes, J.A., Chi, A.R., Cutler, L.S., Healey, D.J., Leeson, D.B., McGunigal, T.E., Mullen Jr., J.A., Smith, W.L., Sydnor, R.L., Vessot, R.F.C. and Winkler, G.M.R. (1971) Characterization of Frequency Stability. IEEE Transactions on Instrumentation and Measurement, IM-20, 105-120.

[7] Allan, D.W. (1966) Statistics of Atomic Frequency Standards. Proceedings of the IEEE, 54, 221-230. http://dx.doi.org/10.1109/PROC.1966.4634

[8] IEEE Standard 1139.

[9] Linear Technology Corporation (2014) LTspice for Windows. http://www.linear-tech.co.jp/designtools/software/ 
Scientific Research Publishing (SCIRP) is one of the largest Open Access journal publishers. It is currently publishing more than 200 open access, online, peer-reviewed journals covering a wide range of academic disciplines. SCIRP serves the worldwide academic communities and contributes to the progress and application of science with its publication.

Other selected journals from SCIRP are listed as below. Submit your manuscript to us via either submit@scirp.org or Online Submission Portal.
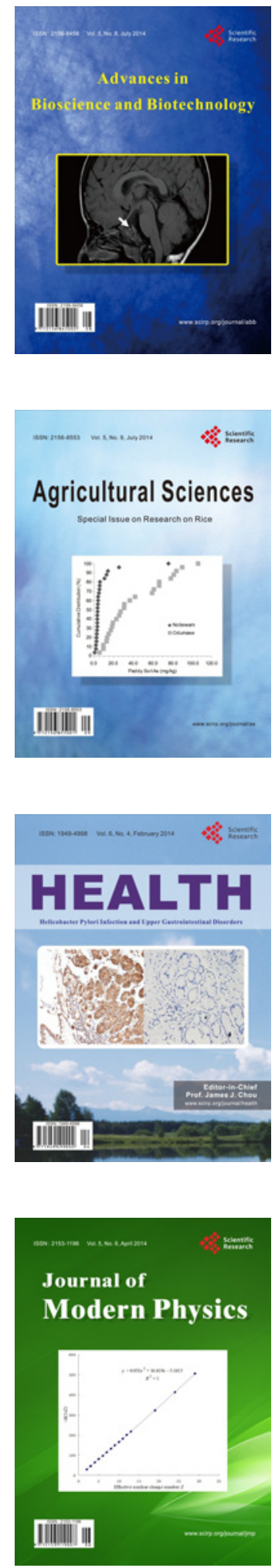
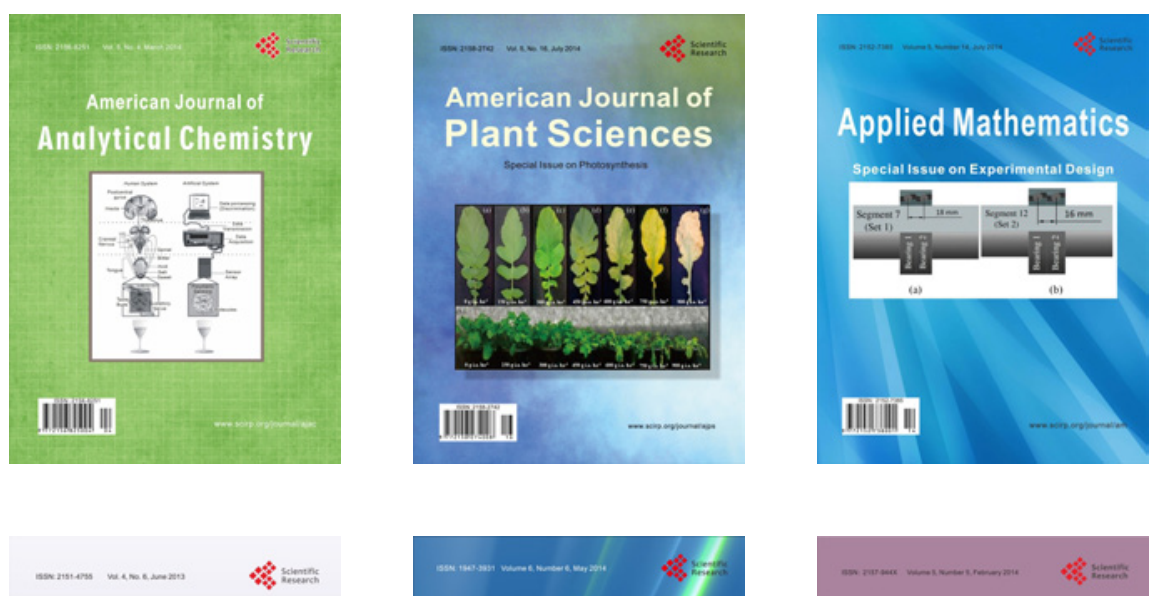

Creative Education
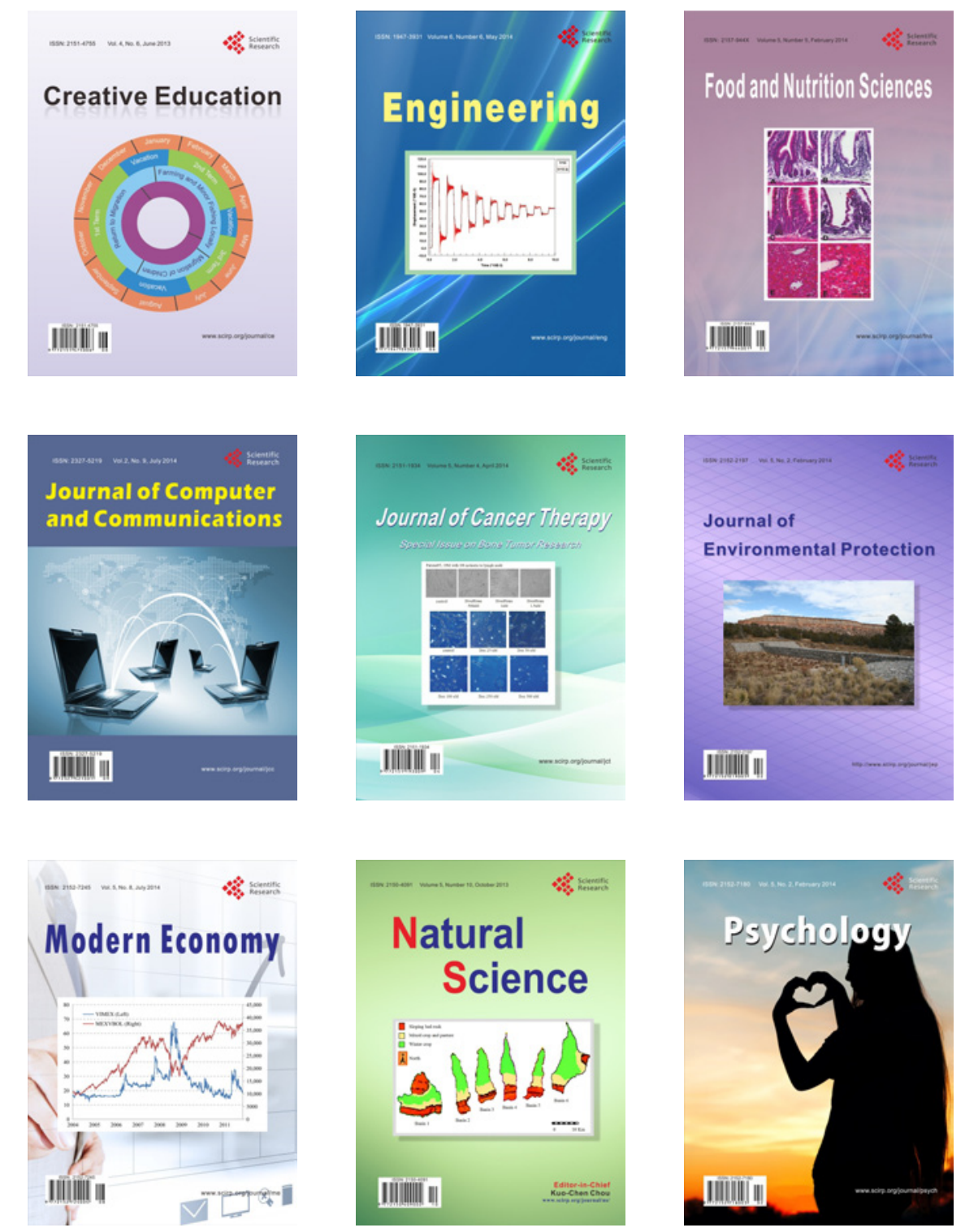